\title{
The effects of supplementary nicotine in regular cigarette smokers
}

\author{
JonATHAN A. MCM. TURNER* \\ M.B.B.S., M.R.C.P.
}

Deanna M. TAYlor

A.S.C.T.

\author{
ROGER W. Sillett \\ M.S.C.T.
}
MARTIN W. MCNiCOL M.B., Ch.B., F.R.C.P.

Cardio-Thoracic Department, Central Middlesex Hospital, London NW10

\begin{abstract}
Summary
Blood nicotine levels were measured in eight subjects over a 5-week period, while smoking normally, while smoking and chewing gum containing 2 mg nicotine, and while smoking and chewing placebo gum. Despite a small but significant rise in blood nicotine levels during the period of the nicotine gum chewing (mean $35.3 \mathrm{ng} / \mathrm{ml}$ ) compared with placebo (mean $28.9 \mathrm{ng} / \mathrm{ml}$ ) and control (mean $26.3 \mathrm{ng} / \mathrm{ml}$ ), cigarette consumption butt lengths, filter nicotine and blood carboxyhaemoglobin levels did not change indicating that there had been no significant changes in smoking patterns.

The reasons for this failure to demonstrate an effect are discussed. It is concluded that the dose of nicotine used was probably not adequate to produce an effect.
\end{abstract}

\section{Introduction}

The role of nicotine in the smoking habit is complex and its importance in the treatment of smoking withdrawal is not fully understood. Conflicting results of its use are reported. Although swallowed nicotine was rapidly metabolized by the liver (Volle and Koell, 1970) large doses $(10 \mathrm{mg} / \mathrm{kg}$ ) have been shown to produce a slight reduction in smoking (Jarvick, Glick and Nakamura, 1970). A trial of aerosol nicotine which is well absorbed (Herxheimer et al., 1967) failed to show any benefit (Chappell, 1974). In acute studies nicotine by injection has been shown to reduce the craving for and consumption of cigarettes (Johnstone, 1942; Lucchesi, Shuster and Emley, 1967). Observations on subjects smoking brands of cigarettes with different nicotine content suggested that they modified smoking patterns in an attempt to regulate nicotine intake (Ashton and Watson, 1970; Frith, 1971; Russell et al., 1975; Turner, Sillett and Ball, 1974). Mecamylamine, which penetrates the central

\footnotetext{
* Present appointment: Sir Jules Thorn Research Fellow, Department of Medicine, Middlesex Hospital, London W1N 8AA.
}

nervous system and is believed to antagonize the action of nicotine, has been shown in an acute $2-\mathrm{hr}$ study to reduce cigarette consumption by $30 \%$ (Stollerman et al., 1973). The wide range of results reported suggest that not only the amount of nicotine but also its route of administration and hence rate of absorption is important. Also the trial design and the circumstances on which observations are made is likely to influence greatly the results. This would not be surprising with a highly conditioned habit such as cigarette smoking.

The authors therefore undertook this study to assess the effect of supplementary nicotine on spontaneous smoking. No attempt was made to influence smoking behaviour and the environment was not that of an anti-smoking clinic. Attempts to produce an effective adminstrable form of nicotine have met with relatively little success. They used a nicotine chewinggum in which nicotine released by chewing is absorbed in part by the buccal mucosa (Ferno, Lichtneckert and Luncren, 1973). It has been shown that this preparation is capable of producing a modest inhibitory effect on smoking behaviour (Russell et al., 1976a).

\section{Subjects and methods}

Eleven healthy members of the hospital staff entered the trial. Eight subjects completed the trial (four men and four women), average age 25 years (range 19-30). The subjects smoked their own brand of filter cigarettes (nicotine $1 \cdot 1-1 \cdot 3 \mathrm{mg}$ ) throughout the trial.

The trial lasted 5 weeks. After a control period of 1 week without gum, the subjects were randomized into two groups: either active gum containing $2 \mathrm{mg}$ nicotine; or placebo gum of identical taste, for 2 weeks followed by the other gum for 2 weeks. The trial was double blind. Subjects were instructed to chew at least ten gums per day, each for $20 \mathrm{~min}$.

They were actively studied in weeks 1,3 and 5 , and venous blood samples were taken at the end of 
each working day and analysed for nicotine (Falkman et al., 1975), and carboxyhaemoglobin (COHb) (Turner et al., 1974).

Subjects recorded on diary cards the number of cigarettes smoked and gums chewed. The time from the last cigarette smoked and from the last gum chewed to the blood sample was recorded.

During each period approximately twenty cigarette butts were collected from each subject and were stored in sealed containers. The length of unburnt tobacco was measured. The cigarette filters were analysed for nicotine content. Similarly, approximately twenty chewed gums were collected from each subject during both gum periods and were analysed for their residual nicotine content (Horwitz, 1960). All results were compared using Student's $t$-test.

\section{Results}

Only eight subjects completed the protocol. One subject felt nauseated when chewing the gum and two other subjects were excluded for technical reasons.

\section{Blood nicotine levels (Table 1)}

There were no significant differences between the three periods in the time of day the blood was taken or the time from the last cigarette or chewing-gum to the venepuncture.
The mean daily blood nicotine levels were control period $26.3 \mathrm{ng} / \mathrm{ml}$, placebo gum $28.9 \mathrm{ng} / \mathrm{ml}$ and nicotine gum $35 \cdot 3 \mathrm{ng} / \mathrm{ml}$. The difference between the control and nicotine periods is significant $(P<0.01)$ as is the difference between the placebo and nicotine periods $(P<0.05)$, but not between the control and placebo periods.

Gum consumption and nicotine extraction (Table 1)

During the nicotine-gum period the average daily gum consumption was eight pieces, five of which were chewed before blood sampling. Analysis of the chewed gums showed a mean residue of $0.95 \mathrm{mg}$ (range 0.622-1.325) nicotine. This indicates a mean daily nicotine extraction from the gum of $8.40 \mathrm{mg}$ (range 4.52-13.78).

Cigarette consumption, butt lengths, filter nicotine and carboxyhaemoglobin (COHb) levels (Table 2)

There was no significant difference in cigarette consumption between the three periods, nor was there any change in butt lengths, filter nicotine content or blood $\mathrm{COHb}$ levels.

\section{Discussion}

Subjects in this trial had significantly raised blood nicotine levels while chewing nicotine-gum but they did not reduce their cigarette consumption or altei

TABLE 1. Changes in blood nicotine, gum consumption, residual nicotine in chewed gums, and nicotine extraction in eight smokers during a control period and while chewing placebo and nicotine gum. Values are mean \pm s.d.

\begin{tabular}{lccc}
\hline & Control & Placebo gum & Nicotine gum \\
\hline $\begin{array}{l}\text { Blood nicotine (ng/ml) } \\
\text { Gum consumption (daily) }\end{array}$ & $26 \cdot 3 \pm 9 \cdot 8$ & $28 \cdot 9 \pm 12 \cdot 0$ & $35 \cdot 3 \pm 8 \cdot 8$ \\
$\begin{array}{l}\text { Gum consumption } \\
\text { (before blood sample) }\end{array}$ & - & $8 \cdot 9 \pm 2 \cdot 4$ & $8 \cdot 0 \pm 2 \cdot 5$ \\
$\begin{array}{l}\text { Residual nicotine in chewed } \\
\text { gums (mg/gum) }\end{array}$ & - & $6 \cdot 1 \pm 2 \cdot 0$ & $5 \cdot 0 \pm 1 \cdot 5$ \\
$\begin{array}{l}\text { Nicotine extraction from gums } \\
\text { (mg/day) }\end{array}$ & - & - & $0 \cdot 95 \pm 3 \cdot 0$ \\
\hline
\end{tabular}

TABLE 2. Changes in cigarette consumption, butt lengths, filter nicotine content and $\mathrm{COHb}$ levels in eight smokers during a control period and while chewing placebo and nicotine gum. Values are mean \pm s.d.

\begin{tabular}{lccc}
\hline & Control & Placebo gum & Nicotine gum \\
\hline $\begin{array}{l}\text { Cigarette consumption (daily) } \\
\text { Cigarette consumption before }\end{array}$ & $21 \cdot 7 \pm 6 \cdot 5$ & $22 \cdot 2 \pm 8 \cdot 6$ & $22 \cdot 4 \pm 7 \cdot 2$ \\
blood sampling & $9 \cdot 4 \pm 3 \cdot 9$ & $10 \cdot 0 \pm 8 \cdot 1$ & $9 \cdot 0 \pm 5 \cdot 5$ \\
Butt length (mm) & $8 \cdot 8 \pm 3 \cdot 8$ & $9 \cdot 1 \pm 3 \cdot 4$ & $7 \cdot 9 \pm 2 \cdot 8$ \\
Filter nicotine content (mg) & $0.99 \pm 0 \cdot 42$ & $0 \cdot 98 \pm 0 \cdot 52$ & $1 \cdot 04 \pm 0 \cdot 48$ \\
COHb \% & $6 \cdot 5 \pm 3 \cdot 1$ & $6 \cdot 3 \pm 3 \cdot 1$ & $6 \cdot 5 \pm 2 \cdot 9$ \\
\hline
\end{tabular}


their inhalation patterns as indicated by $\mathrm{COHb}$ levels or leave longer butts. These results are unexpected since it is generally believed that smokers regulate their smoking behaviour to maintain a steady blood nicotine level. There are three possible explanations for these findings. Firstly, although the blood nicotine levels were elevated, the total nicotine dose given by the chewing-gum was small, possibly insufficient to influence cigarette consumption and inhalation patterns. Secondly, the nicotine chewinggum produces a very different pattern of blood nicotine levels from that following smoking. Finally, it is possible that the circumstances of this trial were sufficiently different from the circumstances of the observations reported by others, and therefore, it is not surprising that a different result was obtained.

Lucchesi, Shuster and Emley (1967) showed that nicotine infusion at the rate of $1 \mathrm{mg} / \mathrm{hr}$ did not significantly alter cigarette consumption although the effect was demonstrated at higher dose levels. Information of the pharmokinetics of nicotine is incomplete and their paper has no information on blood levels. It is probable that $1 \mathrm{mg} / \mathrm{hr}$ of nicotine intravenously produces significantly greater elevation of blood nicotine levels than was produced by the nicotine chewing gum in the present study. In this study the total nicotine dose from the gum is low, subjects extracted on the average $53 \%(1.05 \mathrm{mg})$ of the nicotine in the gums. Of this only about $33 \%(0 \cdot 3$ $\mathrm{mg}$ ) is absorbed in the buccal mucosa, the remainder being swallowed and metabolized without having a significant effect. The daily nicotine intake from the eight gums chewed by the subjects was on average $2.5 \mathrm{mg}$ and although this produced a significant elevation in mean blood nicotine levels, it is well below the daily nicotine intake of most smokers who absorb about $1 \mathrm{mg}$ from each cigarette smoked, and certainly was only a very small proportion as addition to the nicotine dose that the subjects on this trial obtained from the cigarettes they smoked (about $22 \mathrm{mg}$ daily). The total nicotine dose given by the gum to these subjects in the 24-hr period was significantly less than the dose which Lucchesi et al. (1967) found to influence cigarette consumption.

It has been suggested that the pattern of change in blood nicotine levels is also significant. Cigarette smoking with alveolar absorption of nicotine produces blood levels with a very rapid rise to high peak which subsequently falls and on equilibration lower levels are reached (Russell et al., 1976b; Armitage et al., 1975). It is not clear whether the peak levels or the mean values are of importance. With buccal absorption of nicotine a high peak is not produced and the build-up to mean value takes place more slowly over a longer period of time (Russell et al., 1976). This difference in pattern may be important but there is as yet inadequate information to assess its significance.

Finally, one must consider the possibility of environmental influence. The authors' findings are in contrast with those working from anti-smoking clinics (Russell, Feyerabend and Cole, 1970; Brantmark, Ohlim and Westling, 1973; Westling, 1976), who showed that the chewing gum containing nicotine had a clear though modest effect on smoking behaviour. However, the specific contribution of nicotine in the gums was small and by far the greatest effect demonstrated was attributable to the "placebo effect' of the control gum. The authors believe that these changes may be in part attributable to the environment of the clinic in which the study was carried out. Their subjects were all volunteers who had no desire to stop smoking and, although they attended the laboratory in a department which was known to engage in anti-smoking research, the environment was not that of an anti-smoking clinic. It is suggested that this may be a significant fact.

The authors' lack of influence on spontaneous smoking patterns despite a modest rise in blood nicotine levels suggests that a larger nicotine dose will be required in the chewing gums if they are to be effective. Dose is limited by gastro-intestinal intolerance, one of the subjects developed nausea and was unable to complete the trial. The manufacturers have now introduced a $4 \mathrm{mg}$ gum with an improved flavour which may overcome this problem and deliver a greater nicotine dose. Clearly, further studies with a gum of this sort are required to elucidate the role of nicotine in influencing the smoking pattern.

\section{Acknowledgments}

We wish to thank all our subjects who took part in the trial and to Dr K. P. Ball for his advice. We are indebted to A. B. Leo and Company for providing the nicotine chewinggum and for the nicotine analyses.

\section{References}

Armitage, A.K., Dollery, C.T., George, C.F., Houseman, T.H., LEWIS, P.J. \& TURNER, D.M. (1975) Absorption of nicotine from cigarettes. British Medical Journal, 4, 313.

Ashton, H. \& WATson, D.W. (1970) Puffing frequency and nicotine intake in cigarette smokers. British Medical Journal, 3, 679.

Brantmark, B., Ohlim, P. \& Westling, H. (1973) Nicotinecontaining chewing gum as an anti-smoking aid. Psychopharmacologica, 31, 191.

Chappell, G. (1974) Nicotine aerosols in the treatment of cigarette dependence. Proceedings of the British Thoracic and Tuberculosis Association, 29th March.

Falkman, S.E., Burrows, I.E., Lungren, R.W., \& Page, B.F.J. (1975) A modified procedure for the determination of nicotine in blood. Analyst, 100, 99.

Ferno, O., LiChTNeCKeRT, S.J.A. \& LunCREN, C.E.G. (1973) A substitute for tobacco smoking. Psychopharmacologica, 31 (3), 201. 
FrITH, C.D. (1971) The effect of varying nicotine content of cigarettes on human smoking behaviour. Psychopharmacologica, 19, 188.

Herxheimer, A., Griffiths, R.L., Hamilton, B. \& WakeFIELD, M. (1967) Circulatory effects of nicotine aerosol inhalations and cigarette smoking in man. Lancet, ii, 754.

HoRwitz, W. (1960) Official Methods of Analysis of the Association of Analytical Chemists. 9th Edn, pp. 94-95. Washington DC.

Jarvick, M.E., Glick, S.D. \& NaKamura, R.K. (1970) Inhibition of cigarette smoking by orally administered nicotine. Clinical Pharmacology and Therapeutics, 11 (4), 544.

Johnstone, L.M. (1942) Tobacco smoking and nicotine. Lancet, ii, 742.

LuCChesi, B.R., Shuster, C.R. \& EMery, G.S. (1967) The role of nicotine as a determinant of cigarette smoking frequency in man with observations of certain cardiovascular effects associated with the tobacco alkaloid. Clinical Pharmacology and Therapeutics, 8, 789.

Russell, M.A.H., Feyerabend, C. \& Cole, P.V. (1976b
Plasma nicotine levels after cigarette smoking and chewing nicotine gum. British Medical Journal, 1, 1043.

Russell, M.A.H., Wilson, C., Feyerabend, C. \& Cole, P.V. (1976a) Effect of nicotine chewing gum on smoking behaviour and as an aid to cigarette withdrawal. British Medical Journal, 2, 391.

Russell, M.A.H., Wilson, C., Patel, U.A., Feyerabend, C. \& Cole, P.V. (1975) Plasma nicotine levels after smoking cigarettes with high, medium and low nicotine levels. British Medical Journal, 2, 414.

Stollerman, I.P., Goldfarb, T., Fink, R. \& JaRvik, M.E. (1973) Influencing cigarette smoking with nicotine antagonists. Psychopharmacologica, 28, 247.

TuRNer, J.A.McM., Sillett, R.W. \& Ball, K.P. (1974) Some effects of changing to low-tar, low-nicotine cigarettes. Lancet, ii, 737.

Volle, R.L. \& Koell, G.B. (1970) Ganglion stimulating and blocking agents. In: The Pharmacological Basis of Therapeutics (Ed. by L. S. Godman and A. Gilman), 5th Edn, pp. 567-570. MacMillan, New York.

WESTLING, H. (1976) Experiences of nicotine chewing gum in an anti-smoking clinic. Lakartidningen, 73 (30-31), 2549. 